\title{
4
}

\section{Standard Fuzzy Sets and some Many-Valued Logics}

\author{
Jorma K. Mattila \\ Lappeenranta University of Technology \\ Finland
}

\section{Introduction}

The aim of this chapter is to consider the relationship between standard fuzzy set theory and some many-valued logics. Prof. Lotfi A. Zadeh introduced his theory of fuzzy sets in sixties, and his first paper that circulated widely around the world is "Fuzzy Sets" (Zadeh, 1965). In the long run, this theory was began to call by the name theory of standard fuzzy sets.

After Zadeh has introduced his theory, many-valued logic began to have a new interest. Especially, Łukasiewicz logic was enclosed quite closely in fuzzy sets. There is a strong opinion that Łukasiewicz infinite-valued logic has the role as the logic of fuzzy sets, similarly as classical logic has the role as the logic of crisp sets. But actually, it seems that Kleene's 3-valued logic was the closest logic connecting to fuzzy sets, when Zadeh created his theory. We will discuss this thing later. In the books Rescher (Rescher, 1969) and Bergmann (Bergmann, 2008) descriptions about Kleene's logic are given.

In Section 2 we consider the main concepts of fuzzy set theory. We will not do it completely, because our purpose is not to present the whole theory of standard fuzzy sets. We restrict our consideration on those things we need when we are "building a bridge" between fuzzy sets and some closely related logics. The section is based on Zadeh (Zadeh, 1965).

In Section 3 we consider De Morgan algebras in general in order to have a formal base to our consideration. There are many sources for this topic. One remarkable one is Rasiowa's book (Rasiowa, 1974).

In Section 4 we introduce an algebraic approach for standard fuzzy set theory by applying De Morgan algebras. We choose an algebra from the infinite large collection of De Morgan algebras that fits completely to standard fuzzy set theory. We call this De Morgan algebra by the name Zadeh algebra. The concept "Zadeh algebra" was introduced by the author in an international symposium "Fuzziness in Finland" in 2004. Also Prof. Zadeh attended this event. In the same year, a more comprehensive article about Zahed algebra (cf. (Mattila, 2004)) was published by the author. This algebra gives a tool for studying connections between standard fuzzy sets and certain many-valued logics. Two of these logics are Kleene's logic and Łukasiewicz logic. Some analysis about Łukasiewicz and Kleene's logic is given for example in Mattila (Mattila, 2009). Especially, connections to modal logic are considered in that paper. 
In Section 5 we make some analysis about the essence of fuzziness from the formal point of view. We try to find the original point where fuzziness appears and how it "moves" from its hiding-place making some concepts fuzzy.

In Section 6 we give the definition of propositional language by introducing its alphabet and how the expressions, i.e., wellformed formulas (or formulas, for short) can be formed from the alphabet. This formal language can be used as classical propositional logic and as many-valued propositional logic, too. We do not consider any other logical properties here, because they are not necessary for our purpose. In addition to the formal language, only the concept valuation and truth-function are needed. About the truth value evaluation, we consider the common things for several logics. The counterparts are obtainable also from Zadeh algebra. We also construct a propositional algebra that appears to be a Zadeh algebra.

In Section 7 an important logic for fuzzy sets is Kleene's 3-valued logic, as we already noticed above. Hence, the consideration of this logic deserves its own section. We tell about Kleene's motivation for constructing his 3-valued logic and give the truth value evaluation rules for the basic connectives. These rules fit completely well to the fuzzy set operations Zadeh introduced. We also explain the connections between standard fuzzy sets and this logic from Zadeh's point of view. In the end of this section, we give a short description about Kleene-Dienes many-valued logic that is an extension of Kleene's 3-valued logic into infinite-valued logic.

In Section 8 we consider the main features of Łukasiewicz ifinite-valued logic. Our main problem is included in this section. Łukasiewicz chose the connectives negation and implication as primitive connectives and derived the connectives conjunction, disjunction, and equivalence from these primitives. This starting point does not fit together with the operations of Zadeh algebra. Only the counterpart of negation (the complementarity operation) is included in Zadeh algebra but implication does not appear in it. in Łukasiewicz logic the two other connectives, disjunction and conjunction, belongs to the derived connectives. But they have such a form that their truth value evaluation rules are exactly the same as the corresponding operations in Zadeh algebra. So, using the set negation, disjunction, and conjunction of Łukasiewicz logic's connectives, we have to derive the connective Łukasiewicz implication. Actually, for this task we need only negation and disjunction, as is seen in Proposition 8.2 and its proof. Our final result is presented in Proposition 8.3. Some considerations on this topic can be found in Mattila (Mattila, 2005).

In Section 9 we consider briefly MV-algebras and give some hints how the connection between standard fuzzy sets and Łukasiewicz logic can be found. MV-algebras and their applications to fuzzy set theory and soft computing are widely studied, and the study of this topic actually forms a mainstream in this research area. Three books are mentioned in References representing this topic, namely M. Bergmann (Bergmann, 2008), R. L. O. Cignoli et al. (Cignoli et al., 2000), and P. Hájek (Hájek, 1998). These books belongs to a quite central literature of the topic.

MV-algebras are more general than De Morgan algebras, but formally it can be proved that De Morgan algebras belong to MV-algebras as a special case. But according to our problem, the used ways to apply general MV-algebras seems to give a circuitous route rather than a straightforward bridge between standard fuzzy set theory and Łukasiewicz logic.

In Section 10 we point out the main results and other concluding remarks. 


\section{Zadeh's theory of standard fuzzy sets}

For considering the standard system of fuzzy sets, the range of fuzzy sets (i.e., that of membership functions) is the unit interval $\mathbb{I}=[0,1]$. We give the definition of the concept fuzzy set using Zadeh's original definition. However, some symbols have been changed. Usually, the symbol of a fuzzy set, in general, is denoted by $\mu$. A membership function of a fuzzy set $A$ in a reference set $X$ can be written as $\mu_{A}(x)$ or $\mathcal{A}(x)$ where $x \in X$.

Definition 2.1 (Standard fuzzy set). A fuzzy subset $A$ of a set $X$ is characterized by a membership function $\mathcal{A}(x)$ which associates with each point $x$ in $X$ a real number in the interval $[0,1]$, with the value of $\mathcal{A}(x)$ at $x$ representing the "grade of membership" of $x$ in $A$. Thus, the nearer the value of $\mathcal{A}(x)$ to unity, the higher the grade of membership of $x$ in $A$.

This definition means that a fuzzy subset $A$ of a universe of discourse $X$ is represented by a function

$$
\mathcal{A}: X \longrightarrow \mathbb{I} \text {. }
$$

The power set of all fuzzy subsets of the set $X$ is

$$
\mathbb{I}^{X}=\{\mathcal{A} \mid \mathcal{A}: X \longrightarrow \mathbb{I}\}
$$

An important subset of the set of all membership functions (2.1) is the set of functions taking only values 1 or 0 , i.e., the set of all characteristic functions of the crisp subsets of $X$

$$
\mathbf{2}^{X}=\{f \mid f: X \longrightarrow\{0,1\}\}
$$

as a special case.

It is also a well-known fact that II and $\mathbb{I}^{X}$ are partially ordered sets. (Actually, II is a totally ordered set, but hence it is also prtially ordered.) In fact, they are also distributive complete lattices. Generally, some main properties of $\mathbb{I}$ can be embedded to $\mathbb{I}^{X}$ (cf. e.g. Lowen (Lowen, 1996)).

We consider operations, properties, and some concepts involved in fuzzy sets given by Zadeh (Zadeh, 1965).

Definition 2.2 (Basic operations). Let $\mathcal{A}, \mathcal{B} \in \mathbb{I}^{X}$ and $x \in X$. In $\mathbb{I}^{X}$ there are defined the following operations:

$$
\begin{aligned}
(\mathcal{A} \vee \mathcal{B})(x) & =\max \{\mathcal{A}(x), \mathcal{B}(x)\} & & \text { union } \\
(\mathcal{A} \wedge \mathcal{B})(x) & =\min \{\mathcal{A}(x), \mathcal{B}(x)\} & & \text { intersection } \\
\overline{\mathcal{A}}(x) & =1-\mathcal{A}(x) & & \text { complementarity }
\end{aligned}
$$

Two fuzzy sets $\mathcal{A}, \mathcal{B} \in \mathbb{I}^{X}$ are equal, denoted by $\mathcal{A}=\mathcal{B}$, if

$$
\forall x \in X, \quad \mathcal{A}(x)=\mathcal{B}(x) .
$$

A fuzzy set $A$ is contained in a fuzzy set $B$, i.e., $A$ is a subset of $B$, denoted by $\mathcal{A} \subseteq \mathcal{B}$, if their membership functions satisfy the condition

$$
\forall x \in X, \quad \mathcal{A}(x) \leq \mathcal{B}(x)
$$


Zadeh also shows that the operations max and min are associative, distributive to each other, and De Morgan's laws hold, and they have the form

$$
\begin{aligned}
& 1-\min \{\mathcal{A}(x), \mathcal{B}(x)\}=\max \{1-\mathcal{A}(x), 1-\mathcal{B}(x)\} \\
& 1-\max \{\mathcal{A}(x), \mathcal{B}(x)\}=\min \{1-\mathcal{A}(x), 1-\mathcal{B}(x)\}
\end{aligned}
$$

Actually, Zadeh gives the building materials for an algebra in his paper (Zadeh, 1965). However, he did not think any algebras when he created his paper "Fuzzy Sets". He thought the problem from another point of view. We return to this matter in the end of Section 4.

Finally, we present the following theorem due to C. V. Negoită and D. A. Ralescu (Negoită \& Ralescu, 1975).

Theorem 2.1. The set $\mathbb{I}^{X}$ is a complete distributive lattice.

Proof. The reference set $X$ has the membership function

$$
\mu_{X}(x)=1, \quad x \in X
$$

and the empty set $\varnothing$ the membership function

$$
\mu_{\varnothing}(x)=0, \quad x \in X
$$

This corresponds to the fact that $\mathbf{1 , 0} \in \mathbb{I}^{X}$ where $\mathbf{1}(x)=1$ and $\mathbf{0}(x)=0$ for any $x \in X$. Hence, the result follows by the definition of complete lattice and the order properties of the unit interval.

\section{On De Morgan algebras}

To get an algebra of standard fuzzy sets we start by considering the concept of De Morgan algebras. The main source is Helena Rasiowa's book (Rasiowa, 1974).

Definition 3.1 (De Morgan algebra). An abstract algebra $\mathcal{A}=\langle A, \vee, \wedge, \neg, \mathbf{1}\rangle$ is called De Morgan algebra, if $(A, \vee, \wedge)$ is a distributive lattice with unit element 1 (the neutral element of $\wedge$ operation), and $\neg$ is a unary operation on $A$ satisfying the following conditions:

(DM1) for all $a \in A, \quad \neg \neg a=a$,

(DM2) for all $a, b \in A, \quad \neg(a \vee b)=\neg a \wedge \neg b$.

It is easy to prove that in any De Morgan algebra $\langle A, \vee, \wedge, \neg, \mathbf{1}\rangle$ the following properties hold:

(DM3) there is a zero element $\mathbf{0}$ (the neutral element of $\vee$ operation),

(DM4) $\neg \mathbf{0}=\mathbf{1}$ and $\neg \mathbf{1}=\mathbf{0}$,

(DM5) $\neg(a \wedge b)=\neg a \vee \neg b$.

The unit element is the greatest element and the zero element the least element of $A$. By (DM3), we sometimes add the zero element of a De Morgan algebra into the component list of the entities belonging to the algebra: $\mathcal{A}=\langle A, \vee, \wedge, \neg, \mathbf{0}, \mathbf{1}\rangle$. 
Consider the unit interval lattice $\mathbb{I}=([0,1], \leq)$. Sometimes we write $\mathbb{I}=[0,1]$, for short. As is well known, the order relation $\leq$ and the operations $\vee$ and $\wedge$ have the connection

$$
\forall x, y \in X, x \leq y \Longleftrightarrow\left\{\begin{array}{l}
x \vee y=y \\
x \wedge y=x
\end{array}\right.
$$

Hence, we can write the lattice $\mathbb{I}$ into the form $\mathbb{I}=([0,1], \vee, \wedge)$. We will prove it is a distributive lattice. We consider it in the proof of Theorem 3.1 when we prove that II forms a De Morgan algebra. Especially, the order relation $\leq$ is a total order on $[0,1]$ because it is an order and any two elements from the interval $[0,1]$ are comparable with each other under it, i.e., for any $x, y \in \mathbb{I}$, we can state whether the order $x \leq y$ either holds or not.

The interval $[0,1]$ is a metric space with the natural metric distance between two points of $[0,1]$ given by the condition

$$
d(x, y)=|x-y|, \quad x, y \in[0,1]
$$

We will see that this equality measure can be used in Łukasiewicz infinite-valued logic as the evaluation rule for the connective equivalency.

Theorem 3.1. The system $L_{\mathbb{I}}=\langle\mathbb{I}, \vee, \wedge, \neg, 0,1\rangle$ is De Morgan algebra, where for all $x \in[0,1]$, $\neg x=1-x$.

Proof. First, we show that II is a distributive lattice. It is clear that II is a lattice. For showing distributivity, we choose arbitrarily elements $a, b, c \in[0,1]$. Without loss of generality, we can suppose that $a \leq b \leq c$. Then, by (3.1) we have

$$
\left\{\begin{array}{l}
a \vee(b \wedge c)=a \vee b=b \\
(a \vee b) \wedge(a \vee c)=b \wedge c=b
\end{array} \quad \Longrightarrow \quad a \vee(b \wedge c)=(a \vee b) \wedge(a \vee c)\right.
$$

Similarly, we have $a \wedge(b \vee c)=(a \wedge b) \vee(a \wedge c)$. Hence, $\mathbb{I}=([0,1], \vee, \wedge)$ is a distributive lattice.

(DM1) holds because for all $a \in[0,1]$,

$$
\neg \neg a=1-(1-a)=a
$$

(DM2) holds because for all $a, b \in[0,1]$,

$$
\left\{\begin{array}{ll}
\neg(a \vee b)=1-(a \vee b)=1-b & \text { if } a \leq b \\
\neg a \wedge \neg b=(1-a) \wedge(1-b)=1-b & \text { if } a \leq b
\end{array} \quad \Longrightarrow \quad \neg(a \vee b)=\neg a \wedge \neg b\right.
$$

Hence, by Def. 3.1, $L_{\mathbb{I}}$ is a De Morgan algebra.

From the ordering property (3.1) it follows that for all $x, y \in \mathbb{I}$

$$
\begin{aligned}
& x \vee y=\max \{x, y\} \\
& x \wedge y=\min \{x, y\}
\end{aligned}
$$

Hence, we can express the algebra of Theorem 3.1 in the form

$$
L_{\mathbb{I}}=\langle\mathbb{I}, \max , \min , \neg, 0,1\rangle
$$


Let $X$ be a nonempty set. Consider a set of functions $\mu: X \longrightarrow \mathbb{I}$, i.e., the function set

$$
\mathbb{I}^{X}=\{\mu \mid \mu: X \longrightarrow \mathbb{I}\}
$$

We extend the algebra of Theorem 3.1 into an algebra of functions (3.6)

$$
L_{\mathbb{I}^{X}}=\left\langle\mathbb{I}^{X}, \vee, \wedge, \neg, \mathbf{0}, \mathbf{1}\right\rangle
$$

by pointwise calculation. Here $\mathbf{0}$ and $\mathbf{1}$ are constant functions, such that

$$
\forall x \in X, \quad \mathbf{0}: x \mapsto 0, \quad \mathbf{1}: x \mapsto 1
$$

The algebra (3.7) is a De Morgan algebra by its construction. This means that we calculate expressions $\mu(x) \vee v(x), \mu(x) \wedge v(x), \neg \mu(x)$ etc. pointwise for any $x \in X$. Hence, the formulas (3.3) and (3.4) are applicable also in the function algebra (3.7).

As a special case, the algebra (3.7) has a subalgebra

$$
L_{\{0,1\}^{X}}=\left\langle\{0,1\}^{X}, \max , \min , \neg, \mathbf{0 , 1}\right\rangle
$$

being an algebra of characteristic functions of classical sets, $f: X \longrightarrow\{0,1\}$. Sometimes we write 2 instead of $\{0,1\}$, so, especially,

$$
\mathbf{2}^{X}=\left\{f_{A} \mid f_{A}: X \longrightarrow\{0,1\}, A \subset X\right\}
$$

is the classical power set of a set $X$ expressed by characteristic functions. The characteristic function of a given set $A \subset X, f_{A}$, is the function

$$
f_{A}(x)=\left\{\begin{array}{lll}
1 & \text { if } & x \in A, \\
0 & \text { if } & x \notin A
\end{array}\right.
$$

This function indicates by the value $f_{A}(x)=1$ that the element $x \in X$ is an element of $A$ and all the elements of $X$ having the value $f_{A}(x)=0$ are elements of the complement of $A$. As a subalgebra of the algebra (3.7), the algebra (3.9) is a special De Morgan algebra, namely a Boolean algebra.

\section{Algebra of standard fuzzy sets}

Consider the algebra (3.7). We may give a new label to it and use operation symbols max and min instead of $\vee$ and $\wedge$, respectively, by the formulas (3.3) and (3.4). Hence, we have

$$
\mathcal{Z}_{\aleph_{1}}=\left\langle\mathbb{I}^{X}, \max , \min , \neg, \mathbf{0}, \mathbf{1}\right\rangle
$$

The subscript $\aleph_{1}$ means the cardinality of continuum, so, $\mathbb{I}^{X}$ is a continuum because $\mathbb{I}$ is continuum, too. For short, we may refer to $\mathcal{Z}_{\aleph_{1}}$ by $\mathcal{Z}$, without the subscript, if there is no possibility for confusion. The complementarity operation $\neg$ is a mapping

$$
\neg: \mathbb{I}^{X} \longrightarrow \mathbb{I}^{X}, \mu \mapsto \mathbf{1}-\mu
$$

Hence, the complement function of a function $\mu$ is $\mathbf{1}-\mu$, such that for all $x \in X,(\mathbf{1}-\mu)(x)=$ $\mathbf{1}(x)-\mu(x)=1-\mu(x)$. (The proof, that $\neg$ defined in this way is really a membership function, 
is given in the proof of Theorem 4.1.) This thing is analogous to the classical set complement expressed by subtraction a set $A$ to be complemented from the universe of discourse $X$, i.e., $A^{c}=X \backslash A$ where $A \subset X$ and $A^{c}$ is the complement of $A$.

The operations max and min are clearly commutative. Based on the fact that the algebra (3.7) is De Morgan algebra, the algebra (4.1) is De Morgan algebra, too. We call this algebra Zadeh algebra because it is an algebraic description of standard fuzzy set theory, similarly as in classical set theory, a certain Boolean algebra (set algebra or algebra of characteristic functions) is the algebraic description of the system of classical sets. Now, we have the following

Theorem 4.1. Zadeh algebra $\mathcal{Z}=\left\langle\mathbb{I}^{X}, \max , \min , \neg, \mathbf{0}, \mathbf{1}\right\rangle$ is an algebraic approach to standard fuzzy set theory.

Proof.

(i) The operations max and min are exactly the same as in Zadeh's theory by Def. 2.2.

(ii) The operations max and $\min$ are commutative and associative on $\mathbb{I}^{X}$, i.e.,

$$
\max \{\mu, \nu\}=\max \{v, \mu\} \text { and } \max \{\mu, \max \{v, \tau\}\}=\max \{\max \{\mu, \nu\}, \tau\}
$$

for all $\mu, v, \tau \in \mathbb{I}^{X}$ because these laws clearly hold for the elements of $\mathbb{I}$, and these laws can be embedded to $\mathbb{I}^{X}$ by pointwice calculation of values of the functions $\mu \in \mathbb{I}^{X}$ (cf. Lowen (Lowen, 1996)). The same properties hold for min, too.

(iii) From Theorem 2.1, distributive laws follows for $\max$ and $\min$ on $\mathbb{I}^{X}$ because $\left(\mathbb{I}^{X}, \max , \min \right)$ is a distributive lattice and Zaheh-algebra (4.1) is De Morgan algebra. Zadeh (Zadeh, 1965) has also proved these laws.

(iv) For all $\mu \in \mathbb{I}^{X}, \max \{\mu, \mathbf{0}\}=\mu$ and $\min \{\mu, \mathbf{1}\}=\mu$, because for any $x \in X$,

$$
(\max \{\mu, \mathbf{0}\})(x)=\max \{\mu(x), \mathbf{0}(x)\}=\max \{\mu(x), 0\}=\mu(x)
$$

Similarly, for any $x \in X$,

$$
(\min \{\mu, \mathbf{0}\})(x)=\mu(x)
$$

(v) For any membership function $\mu \in \mathbb{I}^{X}$, there exists $\neg \mu \in \mathbb{I}^{X}$, such that for any $x \in X$,

$$
(\neg \mu)(x)=(\mathbf{1}-\mu)(x)=\mathbf{1}(x)-\mu(x)=1-\mu(x)
$$

taking values from the unit interval $[0,1]$. Hence, $\neg \mu \in \mathbb{I}^{X}$, and $\neg$ is the complementarity operation of Zadeh's theory.

(vi) Clearly, Zadeh algebra $\mathcal{Z}$ satisfies the condition $0 \neq \mathbf{1}$, by (iv). Hence, $\mathbf{2}^{X} \subset \mathbb{I}^{X}$. The constant functions $\mathbf{0}$ and $\mathbf{1}$ are the zero element and unit element of the algebra.

This competes the proof.

In classical set theory, an element either is or is not an element of a given set. In fuzzy set theory, we have three possibilities: a membership grade of an element in a given fuzzy set equals either to zero or one, or is between them.

For practical use, we may postulate Zadeh algebra by collecting the nevessary properties together. This means that we build Theor. 4.1 again using the main laws and properties like postulates. The result is as follows. 
Proposition 4.1. Let $\mathbb{I}^{X}=\{\mu \mid \mu: X \longrightarrow \mathbb{I}\}$ be the set of all functions from $X$ to $\mathbb{I}$, where the operations $\max$ and $\min$ are pointwise defined between membership functions, and $\neg \mu \stackrel{\text { def }}{=} \mathbf{1}-\mu$. Then $\mathcal{Z}=\left\langle\mathbb{I}^{X}, \max , \min , \neg, \mathbf{0}, \mathbf{1}\right\rangle$ is Zadeh algebra if it satisfies the conditions

$(\mathcal{Z} 1)$ The operations max and min are commutative on $\mathbb{I}^{X}$;

$(\mathcal{Z} 2)$ The operations max and $\min$ are associative on $\mathbb{I}^{X}$;

$(\mathcal{Z} 3)$ The operations max and $\min$ are distributive to each other;

$(\mathcal{Z} 4)$ The neutral elements of the operations $\max$ and $\min$ are $\mathbf{0}$ and $\mathbf{1}$, respectively, i.e., for all $\mu \in \mathbb{I}^{X}$, $\max \{\mu, \mathbf{0}\}=\mu$ and $\min \{\mu, \mathbf{1}\}=\mu$;

$(\mathcal{Z} 5)$ For any membership function $\mu \in \mathbb{I}^{X}$, there exists $\neg \mu \in \mathbb{I}^{X}$, such that $(\neg \mu)=1-\mu$;

$(\mathcal{Z} 6) \quad 0 \neq 1$.

Definition 4.1 (Kleene algebra). De Morgan algebra is Kleene algebra if it satisfies the additional condition

(K) $\quad x \wedge \neg x \leq y \vee \neg y$.

Theorem 4.2. Zadeh algebra (4.1) is a Kleene algebra.

Proof. Zadeh algebra is De Morgan algebra. The condition (K) in Zadeh algebra has the form

$$
\min \{\mu, \neg \mu\} \leq \max \{v, \neg v\}
$$

for all $\mu, v \in \mathbb{I}^{X}$.

To prove this, we can easily show that always $\min \{\mu, \neg \mu\} \leq \frac{1}{2}$ and $\frac{1}{2} \leq \max \{\nu, \neg \nu\}$ for arbitrary $\mu, v \in \mathbb{I}^{X}$, where the result follows immediately.

An alternative way is an easy task to check the four cases: $\left(1^{\circ}\right) \mu \leq \frac{1}{2}, v \leq \frac{1}{2},\left(2^{\circ}\right) \mu \leq \frac{1}{2}, v>$ $\frac{1}{2},\left(3^{\circ}\right) \mu>\frac{1}{2}, v \leq \frac{1}{2}$, and $\left(4^{\circ}\right) \mu>\frac{1}{2}, v>\frac{1}{2}$, and find out that each of these cases satisfies the condition (K).

Zadeh algebra $\mathcal{Z}_{\aleph_{1}}$ has subalgebras which are Zadeh algebras, too. A range of membership functions can be a suitable subset of the unit interval $[0,1]$, such that the postulates of Prop. 4.1 are satisfied. Here the suitability means that the set is closed under the operations of the algebra.

Example 4.1. Consider a set $A=\{0,1\}$ which is a subset of $[0,1]$ consisting of the extreme cases of the unit interval. The algebra $\left\langle A^{X}, \max , \min , \neg, \mathbf{0}, \mathbf{1}\right\rangle$ satisfies the conditions of Zadeh algebra. This algebra is really an extreme case, because it is the Boolean algebra of characteristic functions of strict (i.e., usual) sets. It is a subalgebra of $\mathcal{Z}_{\aleph_{1}}$.

Example 4.2. Consider a set $A=\left\{0, \frac{1}{2}, 1\right\}$ being a subset of $[0,1]$. The set $A$ is the range of functions $\mu: X \longrightarrow A$ where $X \neq \varnothing$ is a set. These functions belong to the set $\mathbb{I}^{X}$, by means of which $A$ is a subset of $\mathbb{I}^{X}$. The conditions of Prop. 4.1 are clearly satisfied. Hence, $\mathcal{Z}_{3}=\left\langle A^{X}\right.$, max, min, $\left.\neg, \mathbf{0}, \mathbf{1}\right\rangle$ is a 3-valued Zadeh algebra, and hence, a subalgebra of $\mathcal{Z}_{\aleph_{1}}$.

Example 4.3. Consider a set $A$ consisting of all the rationals from the unit interval $[0,1]$. The number of the elements of $A$ is countable, but infinite. Hence, the cardinality of $A$ is $\aleph_{0}$. Making similar considerations as in the previous example, we verify that $\mathcal{Z}_{\aleph_{0}}=$ $\left\langle A^{X}, \max , \min , \neg, \mathbf{0}, \mathbf{1}\right\rangle$ is a subalgebra of $\mathcal{Z}_{\aleph_{1}}$. 
Zadeh-algebra as a special case of De Morgan algebras give rise to closer analysis. Here we have done some part of it. The author thinks that Prof. Zadeh did not necessarily think about De Morgan algebras, when he created his crucial paper "Fuzzy Sets" (Zadeh, 1965). He thought the problem from another point of view, as can be seen in the construction of the paper. His leading idea was to model things in the eventful real world. In any way, it was a happy event that Prof. Zadeh's ideas met such a mathematical frame we have considered here. No others have been so successful to find such a right interpretation to some formal tools for modeling real world incidences. In the same time the problem of interpretation of many-valued logic got a solution. Many-valued logic began to give meaningful tools for analyzing and modeling things in real world. The role of many-valued logics were very nominal before Prof. Zadeh invented fuzzy set theory. After this, the study of many-valued logic met a new rise. Fuzzy set theory and fuzzy logic has helped the researchers to find new aspects from already existing mathematical theories. This kind of work is now going on very strongly.

\section{Where is the hiding-place of fuzziness?}

For example, imagine a set of beautiful women. Let us denote this set by $A$. There are women who do not belong to $A$ with the highest grade 1 . So, such a woman does not have some features which would make her beautiful. But she may have some of those features anyway. An intuitive hint about a possible answer to the question: "Where is the hiding-place of fuzziness?" can be found just on the second line above: "... does not ..." It seems that a partial complementarity is somehow involved in this problem.

Let us compare Zadeh algebra with a general Boolean algebra with a supposition that the binary operations are associative because associativity holds in Zadeh algebra. The definition of this kind of Boolean algebra can be postulated as follows.

Definition 5.1. Let $\wedge$ (meet) and $\vee$ (join) be binary operations, and ' (complement) a unary operation on a set $B(\neq \varnothing)$, and let $\mathbf{0}$ and $\mathbf{1}$ be the elements of $B$, such that the following axioms hold:

(BA1) $\wedge$ and $\vee$ are commutative in $B$, i.e., $\forall x, y \in B, x \vee y=y \vee x$ ja $x \wedge y=y \wedge x$;

(BA2) The operations $\wedge$ and $\vee$ are associative in $B$;

(BA3) The operations $\wedge$ and $\vee$ are distributive, i.e.,

$$
\begin{aligned}
\forall x, y, z \in B, \quad & x \wedge(y \vee z)=(x \wedge y) \vee(x \wedge z), \\
& x \vee(y \wedge z)=(x \vee y) \wedge(x \vee z) .
\end{aligned}
$$

(BA4) $\forall x \in B, x \vee \mathbf{0}=x$ and $x \wedge \mathbf{1}=x$, i.e., $\mathbf{0}$ and $\mathbf{1}$ are the neutral elements (or identity elements) of the operations $\vee$ and $\wedge$.

(BA5) For every element $x \in B$ there exists an element $x^{\prime} \in B$, such that $x \vee x^{\prime}=\mathbf{1}$ and $x \wedge x^{\prime}=\mathbf{0}$.

(BA6) For the elements $\mathbf{0}$ and $\mathbf{1}$ of $B$ the condition $\mathbf{0} \neq \mathbf{1}$ holds.

Hence, the set $B$ together with these operations forms a Boolean algebra $\mathcal{B}=\left(B, \vee, \wedge,{ }^{\prime}, \mathbf{0}, \mathbf{1}\right)$.

The only structural difference between these algebras is that between the axioms Z5 and BA 5. BA 5 is characteristic for complement operation, but Z5 does not satisfy the conditions of complement. So, fuzziness lies in the axiom Z5. The influence of this axiom is that also other 
values can be considered as membership degrees than only 0 and 1 . In Boolean algebras with the universe of discourse $\{0,1\}$ the postulate BA5 do not cause conflicts, like the intermediate values may do if these values are added to the universe.

Because complement operation satisfies the conditions of strong negation, a Boolean algebra $\mathcal{B}=\left(\{0,1\}, \vee, \wedge,{ }^{\prime}, \mathbf{0}, \mathbf{1}\right)$ is a special case of $\mathcal{Z}$, i.e., the classical case is included in $\mathcal{Z}$. Trivially, $\{0,1\} \subset[0,1]$. This means that crisp sets are special cases of fuzzy sets, as they should be also according to Zadeh's own theory. See also the proof of Theorem 2.1.

We may conclude that formally the core hiding-place of fuzziness is the statement Z5 in Proposition 4.1. In a concept being fuzzy there is always something that does not hold, i.e., some missing particle the concept does not have. Hence, the complementarity is somehow involved in a fuzzy concept.

\section{Common features of many-valued logics based on Zadeh algebra}

We consider here some preliminary things being common for several many-valued logics. The main purpose is to find a connection between Zaheh algebra and the structures of some many-valued logics. We consider only some propositional logics, because the main concepts we consider here are basic to higher order many-valued logics, too. We restrict our considerations only to structural properties.

First, we need a formal language for our considerations. This language is that of propositional logic.

Definition 6.1. A propositional lanuage $\mathcal{L}$ consists of

1. a set of propositional letters $p_{0}, p_{1}, \ldots, p_{k}, \ldots$ and

2. the truth-functional connectives ' $\wedge$ ', ' $\vee$ ', and ' $\neg$ '.

These symbols are the aplhabets of the propositional language.

Usually only the so-called primitive connectives belong to the alphabet, but it is possible to choose some other connectives to the alphabet, too. Hence, we could drop either conjunction or disjunction from the alphabet if we like. Primitive connectives are connectives from which we can derive the other connectives.

In a standard nonclassical propositional language, the meanings of the connectives ' $\wedge$ ' (conjunction), ' $\vee$ ' (disjunction), and ' $\neg$ ' (negation) can be given as follows: negation $\neg$ is a stong negation (i.e., it is a negation with involution property $\neg \neg p \equiv p$ ), conjunction $\wedge$ is $g l b$ (greatest lower bound), and disjunction $\vee$ is $l u b$ (least upper bound). The symbol ' $\equiv$ ' and $\Longleftrightarrow$ are used as a meta-symbols of equivalency, i.e., this symbol does not belong to the alphabet of the object language which is the language of a formal logic under consideration.

Definition 6.2. Well-formed formulas of $\mathcal{L}$ are given as follows:

$$
\alpha::=p_{k}|\neg \varphi| \varphi \wedge \psi \mid \varphi \vee \psi
$$

In this recursive production system of well formed formulas (wff's, for short) the symbol $p_{k}$ represents any propositional letter and lower case Greek letters are labels of any atomic or connected wff's. Hence, $\alpha$ is a label for any wff, and similarly $\varphi$ and $\psi$ represent any 
propositional wff's. Starting with propositional letters, we can combine them by connectives in the way shown by the production system (6.1). And finally, we can combine any formulas according to the production system.

We can refer to the propositional letters also by lower case letters in general, and to combined formulas by lower case Greek letters or by usual capital letters. These letters belong to the metalanguage we use when we discuss and describe object language. Here we use English equipped by some formal symbols (so-called meta-symbols) as a metalanguage.

The definitions 6.1 and 6.2 above defines the language of propositional logic. Second, we consider some central semantical concepts being necessary for our consideration. This means that we will not present the whole machinery of formal semantics of standard many-valued logics.

We have two important functions, valuation and truth-function we need in our consideration. They are defined as follows.

Definition 6.3. Valuation $v$ is a function

$$
v: \text { Prop } \longrightarrow \mathbb{I}
$$

that associates truth values to propositional letters. Prop is a set of propositional variables.

Truth-function is a function

$$
V_{n}: \mathbb{I}^{n} \longrightarrow \mathbb{I}, \quad n=1,2,3, \ldots,
$$

where $n$ is the number of propositional variables $p, q, \ldots$ in the formula defining a truth function.

In general, truth-functions are functions of several variables defined on the $n$-tuple of the set of truth values, $[0,1]^{n}$ where the independent variables are proposition letters. The subindices $n=1,2,3, \ldots$ are usually dropped. Actually, a propositional formula $\varphi$ itself is a truth-function. Suppose that a formula $\varphi$ consists of the propositional letters $p, q$, and $r$. Then we may write $\varphi=V(p, q, r)$. The equality sign is used only between truth-functions and truth values.

In connected formulas, valuations of propositional variables give the values for the variables of the corresponding truth-function presented by the connected formula. Hence, a "valuation" of a connected formula is the value of the corresponding truth-function. Hence, to evaluate a truth value of the whole connected formula corresponding a given valuation for propositional variables, we calculate the value of the truth-function where the given valuation $v$ first determines the values of the arguments of the truth-function. We may denote the truth value of a connected formula $\varphi$ by $V(\varphi)$, being like a valuation depending on a given valuation $v$ for propositional letters.

Example 6.1. Evaluate the truth value of a formula $p \wedge(\neg q \vee r)$ with regard to a given valuation $v$ for $p, q$, and $r$. Actually the formula is a truth-function $f(p, q, r)=p \wedge(\neg q \vee r)$ where $p, q$, and $r$ obtain their values from $[0,1]$. These values are $v(p), v(q)$, and $v(r)$. Now, the truth value of the formula,given by this valuation $v$, is

$$
V(p \wedge(\neg q \vee r))=v(p) \wedge(\neg v(q) \vee v(r)
$$


Suppose that $v$ is a valuation where $v(p)=0.5, v(q)=0.3$, and $v(r)=1$. Hence,

$$
V(p \wedge(\neg q \vee r))=0.5 \wedge((1-0.3) \vee 1)=0.5 \wedge(0.7 \vee 1)=0.5 \wedge 1=0.5
$$

If we have two wff's representing the same state of affairs we use meta-equivalence sign ' ${ }^{\prime}$ or ' $\Longleftrightarrow$ ' between them because the formulas are equivalent to each other, not identical. For example, the wff $\varphi$ is the formula $p \wedge(\neg q \vee r)$. So, we can write $\varphi \equiv p \wedge(\neg q \vee r)$, or $\varphi \Longleftrightarrow$ $p \wedge(\neg q \vee r)$ to denote that we use an abbreviation $\varphi$ for the formula $p \wedge(\neg q \vee r)$. Another case is that we have two formulas being equivalent to each other, for example, $\neg p \vee \neg q \equiv \neg(p \wedge q)$. This equivalency describes one of De Morgan's laws. However, the expression

$$
\forall x, y \in[0,1], \quad \neg x \vee \neg y=\neg(x \wedge y)
$$

emphasizes that two truth-functions $\neg x \vee \neg y$ and $\neg(x \wedge y)$ are identical.

Instead of propositional letters, we prefer to use "usual" variable symbols as variables of a truth-function, because of possible confusions.

We are interested in the logics where the evaluation rules for these connectives are

$$
\begin{aligned}
V(p \vee q) & =\max \{v(p), v(q)\} \\
V(p \wedge q) & =\min \{v(p), v(q)\} \\
V(\neg p) & =1-v(p)
\end{aligned}
$$

where $v(p), v(q) \in[0,1]$, or $v(p), v(q) \in A$ where $A$ is a suitable subset of $[0,1]$.

Evaluation rules are rules for evaluating truth values to connected logical formulas in a given logic.

We must remember that all the logics are not truth-functional. For example, modal logics are non-truth-functional.

In practice, we need some other connectives, too. Two of them are the connectives implication and equivalency.

The way to choose implication separates the logics based on Zadeh algebra or any De Morgan algebra. Hence, implication must be presented by means of disjunction and negation, or by means of conjunction and negation. There are several ways to define different implications from other connectives, depending on the logic in question. We consider these things in the case of each logic to be considered below.

The formulas (6.4), (6.5), and (6.6) somehow emphasize the relationship to algebraic construction. We have two binary operations and one unary operation defined on a nonempty set just as in usual algebraic system. Additionally, the binary operations are combined together, for example, being distributive. The formulas (6.4), (6.5), and (6.6) are the same as Zadeh's operations defined on the set of fuzzy sets. The bridge between standard fuzzy sets and some many-valued logics seems to be obvious. Having got this kind of motivation, we continue our construction of the bridge between standard fuzzy sets and some many-valued logics.

Consider Zadeh algebra $\mathcal{Z}_{\aleph_{1}}=\left\langle\mathbb{I}^{X}\right.$, max, $\left.\min , \neg, \mathbf{0}, \mathbf{1}\right\rangle$ (cf. formula (4.1)). Now the question is wether there is a counterpart to this algebra in the scope of many-valued logic. According to 
the evaluation rules (6.4), (6.5), and (6.6), the operations $\max$, min, and $\neg$ exist at least in the logics having these evaluation rules. Let us compare the power set of fuzzy sets of the set $X$, i.e., the set $\mathbb{I}^{X}$, to the set of all valuations $v:$ Prop $\longrightarrow \mathbb{I}$. Hence, the set of all valuations is $\mathbb{I}^{\text {Prop }}$. Especially, $\mathbf{1}$ and $\mathbf{0}$ are constant valuations, such that $\mathbf{1}$ gives the truth value 1 to every propositional letters, and similarly, $\mathbf{0}$ gives the truth value 0 . Hence, we also have the neutral elements corresponding those in Zadeh algebra. It seems that if we replace the set $\mathbb{I}^{X}$ by $\mathbb{I}^{\text {Prop }}$ then we have a special Zadeh algebra, namely, say, propositional algebra

$$
\mathcal{L}_{\aleph_{1}}=\left\langle\mathbb{I}^{\text {Prop }}, \max , \min , \neg, \mathbf{0}, \mathbf{1}\right\rangle \text {. }
$$

The values of valuations are truth values and those of membership functions are membership grades. Can these two interpretations for the elements $[0,1]$ be considered to be anyhow similar? According to formal consideration, we say yes. The both values are obtainable from the same set, namely from the unit interval $[0,1]$, and the construction of the both algebras are exactly the same. On the other hand, membership grades are in principle subjective opinions about the membership of an element in a given set. About truth values, a degree of truth of a given propositional letter in a given situation depends on the state of affairs associated to this situation. But there is a valuation for every state of affairs in any situation representing a suitable degree of truth expressed by a number obtained from $[0,1]$. Hence, these degrees of truth correspond to suitable membership grades even so that for any valuation there exists a membership function that is identical with the valuation. Hence, these two apparently different interpretations can be considered to be the same. This means that we can interpret the values of the functions of the algebra (6.7) as truth values, or more accurately, degrees of truth.

\section{Description of Kleene's logic}

For historical reasons, we consider a piece of Kleene's 3-valued logic. S. C. Kleene was Zadeh's logic teacher, and it is natural that Zadeh compared his concept of fuzzy set with Kleene's 3-valued logic. Zadeh ((Zadeh, 1965) p. 341-342) gives the following comment:

"Note that the notion of belonging", which plays a fundamental role in the case of ordinary sets, does not have the same role in the case of fuzzy sets. Thus, it is not meaningful to speak of a point $x$ "belonging" to a fuzzy set $A$ except in the trivial sense of $f_{A}(x)$ being positive. Less trivially, one can introduce two levels $\alpha$ and $\beta$ $(0<\alpha<1,0<\beta<1, \alpha>\beta)$ and agree to say that (1) " $x$ belongs to $A$ " if $f_{A}(x) \geq \alpha ;(2)$ " $x$ does not belong to $A$ " if $f_{A}(x) \leq \beta$; and (3) " $x$ has an intermediate status relative to $A$ " if $\beta<f_{A}(x)<\alpha$. This leads to a three-valued logic (Kleene, 1952) with three truth values $T\left(f_{A}(x) \geq \alpha\right), F\left(f_{A}(x) \leq \beta\right)$, and $U\left(\beta<f_{A}(x)<\alpha\right)$.

The symbols of the truth values of Kleene's 3-valued logic are T (true), $U$ (unknown), and $F$ (false). In the literature, there are also some alternative symbols for the intermediate truth value. For example, Rescher (Rescher, 1969) uses the symbol $I$.

Kleene introduced his 3-vaued logic in 1938. We denote it by $\mathbf{K}_{3}$. In order to describe Kleene's logic, we refer to Rescher (Rescher, 1969), p. 34 - 36. He writes:

"In Kleene's system, a proposition is to bear the third truth-value I not for fact-related, ontological reasons but for knowledge-related, epistemological ones: it is not to be excluded that the proposition may in fact be true or false, but it is merely unknown or undeterminable what its specific truth status may be. 
In $\mathbf{K}_{3}$, we have the following truth value evaluation rules for the connectives negation $\neg$, conjunction $\wedge$, and disjunction $\vee$ :

$$
\begin{aligned}
V(\neg p) & =T-v(p), \\
V(p \wedge q) & =\min \{v(p), v(q)\}, \\
V(p \vee q) & =\max \{v(p), v(q)\} .
\end{aligned}
$$

Two of these connectives can form a set of primitive connectives. One of the primitives must be negation. Hence, for example, the connectives negation and disjunction can be chosen as primitives, and all the other connectives can be defined by means of these primitive connectives. The alternative case for primitives are negation and conjunction. Hence, we can define the nonprimitive one by negation and the fact that disjunction and conjunction are dual (i.e., by using a suitable De Morgan's law). The implication defined by means of negation and disjunction is given by the formula (7.4).

We see immediately that there is a strong analogy between the basic operations of fuzzy sets (cf. Def. 2.2) and these three connectives of Kleene's 3-valued logic. In addition to this, an analogy can be found between Kleene's valuations and Zadeh's membership functions, too, although they have different ranges. Zadeh's comment above connects these two concepts "membership" $(\mu: X \longrightarrow \mathbb{I})$ and "valuation" $(v:$ Prop $\longrightarrow\{F, U, T\})$ together. We can compare the set $\{F, U, T\}$ with the set $\left\{0, \frac{1}{2}, 1\right\}$ where $F=0, U=\frac{1}{2}$, and $T=1$. Hence, by analogy of the sets, we can understand some arithmetic operations in some evaluation rules.

Kleene defined the implication of his 3-valued logic, denoted by $\ni$, analogously to material implication:

$$
p \ni q \stackrel{\text { def }}{\Longleftrightarrow} \neg p \vee q,
$$

hence, the evaluation rule of $p \ni q$ is

$$
p \ni q=\max \{T-v(p), v(q)\} .
$$

We may construct the truth tables according to the evaluation rules. Rescher tells that Kleene motivated the construction of his truth tables in terms of a mathematical application. He has in mind the case of a mathematical predicate $P$ (i.e., a propositional function) of a variable $x$ ranging over a domain $D$ where " $P(x)$ " is defined for only a part of this domain. For example, we might have the condition

Here $P(x)$ will be:

$$
P(x) \quad \text { iff } \quad 1 \leq \frac{1}{x} \leq 2
$$

(1) true if $x$ lies within the range from $\frac{1}{2}$ to 1 ,

(2) undefined (or undetermined) if $x=0$,

(3) false in all other cases.

Kleene presented his truth tables to formulate the rules of combination by logical connectives for such propositional functions. He writes:

"From this standpoint, the meaning of $Q \vee R$ is brought out clearly by the statement in words: $Q \vee R$ is true, if $Q$ is true (here nothing is said about $R$ ) or if $R$ is true (similarly); false, if $Q$ and $R$ are both false; defined only in these cases (and hence undefined, otherwise)."1

\footnotetext{
${ }^{1}$ Kleene, Introduction to Metamathematics (1952).
} 
Clearly, the algebraic approach to $\mathbf{K}_{3}$ is Kleene algebra, i.e., a 3-valued Zadeh algebra with the property $(\mathrm{K})$.

Kleene-Dienes many-valued logic:

Kleene-Dienes many-valued logic is an extension of $\mathbf{K}_{3}$ into $\mathbf{K}_{\aleph_{1}}$ having the set of truth values $[0,1]$. The evaluation rules for conjunction, disjunction, and negation are the same as in the formulas (6.4), (6.5), and (6.6) above.

Implication of Kleene-Dienes many-valued logic is defined by

$$
p \rightarrow q \stackrel{\text { def }}{\Longleftrightarrow} \neg p \vee q
$$

being in accordance with the implication of $\mathbf{K}_{3}$. This means that the evaluation rule for implication is as follows. For any $x, y \in[0,1]$,

$$
x \rightarrow y=\max \{1-x, y\}
$$

Now, the connective equivalence is defined in the usual way:

$$
p \leftrightarrow q \stackrel{\text { def }}{\Longleftrightarrow}(p \rightarrow q) \wedge(q \rightarrow p)
$$

Hence, the evaluation rule for equivalence is

$$
\forall x, y \in[0,1], \quad x \leftrightarrow y=\min \{x \rightarrow y, y \rightarrow x\}
$$

The equations (6.4), (6.5), (6.6), (7.7), and (7.9) are the truth value evaluation rules for disjunction, conjunction, negation, implication, and equivalence, respectively, of Kleene-Dienes many-valued logic with the set of truth values $[0,1]$.

The implication operation of Kleene-Dienes many-valued logic is a typical example about a so-called S-implication. Another example is the implication operation of classical logic. The general principle for S-implication is just the formula (7.6).

\section{On Łukasiewicz' many-valued logic}

We begin with Łukasiewicz' many-valued logic $Ł_{\aleph_{1}}$ having the closed unit interval $[0,1]$ as the set of truth values. ${ }^{2}$

As we know, Łukasiewicz chose the connectives of negation and implication as primitives. This is a remarkable difference, for example, between Kleene's logic and Łukasiewicz logic. Hence, the connection between standard fuzzy set theory and $\succeq_{\aleph_{1}}$ cannot be seen immediately.

Let $v$ be any valuation of $\succeq_{\aleph}$, then the truth value evaluation rules for negation and implication are

$$
\begin{aligned}
v(\neg p) & =1-v(p) \\
v(p \rightarrow q) & =\min \{1,1-v(p)+v(q)\}
\end{aligned}
$$

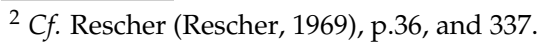


By means of these connectives, Łukasiewicz defined the other connectives by the rules

$$
\begin{aligned}
& p \vee q \stackrel{\text { def }}{\Longleftrightarrow}(p \rightarrow q) \rightarrow q \\
& p \wedge q \stackrel{\text { def }}{\Longleftrightarrow} \neg(\neg p \vee \neg q) \\
& p \leftrightarrow q \stackrel{\text { def }}{\Longleftrightarrow}(p \rightarrow q) \wedge(q \rightarrow p)
\end{aligned}
$$

The truth value evaluation rules for these derived connectives are

$$
\begin{array}{ll}
\max \{v(p), v(q)\} & \text { for } p \vee q, \\
\min \{v(p), v(q)\} & \text { for } p \wedge q, \\
1-|v(p)-v(q)| & \text { for } p \leftrightarrow q
\end{array}
$$

for any valuation $v$ of $€_{\aleph_{1}}$.

In Zadeh algebra we have the operations representing disjunction, conjunction, and negation as given. Negation in Zadeh algebra has the same construction as that in Łukasiewicz' logic $€_{\aleph_{1}}$, so, we need not to do anything with it. Now our task is to derive algebraically the implication of $\epsilon_{\aleph_{1}}$ by means of these three other connectives. For this we use the operations of Zadeh algebra. Actually, we need only complementarity and max operations in our solution. After succeeding to solve this problem we know that standard fuzzy sets and $\bigsqcup_{\aleph_{1}}$ fits together completely, i.e., we can derive all the connectives of $\succeq_{\aleph_{1}}$ in terms of Zadeh algebra. The final result is given in Proposition 8.3. This is the main task in this section.

Consider again the special case of Zadeh algebra (6.7)

$$
\mathcal{L}_{\aleph_{1}}=\left\langle\mathbb{I}^{\text {Prop }}, \max , \min , \neg, \mathbf{0}, \mathbf{1}\right\rangle \text {. }
$$

From the considertations above, we know that

- $\mathcal{L}_{\aleph_{1}}$ is a special Zadeh algebra, namely propositional algebra.

- The binary operations max and min are commutative and associative.

- The operations max and min are distributive to each others.

- The unary operation $\neg$ is a complementarity operation with the property of involution.

We observed in Section 3 that $[0,1]$ is a metric space with the natural metric distance (3.2)

$$
d(x, y)=|x-y|, \quad x, y \in[0,1] .
$$

This formula satisfies the general definition of the concept metric. We need it in the following consideration where we manipulate expressions involving maxima and minima.

In manipulating maxima and minima, the consideration can sometimes be done easier by using the following expressions for max and min operations:

$$
\max \{x, y\}=\frac{x+y+|x-y|}{2}, \quad \min \{x, y\}=\frac{x+y-|x-y|}{2}
$$

These formulas hold on the set of real numbers $\mathbb{R}$, and especially on the unit interval $[0,1]$.

First, consider the case where the operations min and max are used in the form of the formulas (8.4), and $\neg$ is defined in the usual way: $\neg x=1-x$. 
Proposition 8.1. Suppose $x, y \in[0,1]$. Then De Morgan's laws hold for $\min \{x, y\}$ and $\max \{x, y\}$.

Proof. Consider the operation $\min (x, y)$ and $\max (x, y)$, where $x$ and $y$ are variables taking their values from the interval $[0,1]$. Using the arithmetical formula for min operation (i.e., the expression for min in (8.4)), we have

$$
\begin{aligned}
\min \{x, y\} & =\frac{x+y-|x-y|}{2} \\
& =\frac{2-1-1+x+y-|1-1+x-y|}{2} \\
& =1-\frac{1+1-x-y+|1-1+x-y|}{2} \\
& =1-\frac{(1-x)+(1-y)+|(1-y)-(1-x)|}{2} \\
& =1-\max \{(1-x),(1-y)\} .
\end{aligned}
$$

From the formula (8.5), by replacing $x$ by $1-x$ and $y$ by $1-y$, and then solving $\max (x, y)$, the following formula follows:

$$
\max \{x, y\}=1-\min \{1-x, 1-y\}
$$

The formulas (8.5) and (8.6) show that DeMorgan laws hold for max and min, and they are dual of each other.

This completes the proof.

Łukasiewicz knew that the operations max and min are dual of each other. Actually, this property is easily found in the classical special case, i.e. using characteristic functions in presenting crisp sets. But the general proof for this is easily done by using the expressions (8.4) for max and min in such cases where a distance metric is defined in the universe of discourse. This always holds at least for real numbers.

Second, consider the connection between max and Łukasiewicz implication using Zadeh algebra (similar considerations are done in Mattila (Mattila, 2005), but the following proposition 8.2 is not completely proved).

Proposition 8.2. For all $x, y \in[0,1]$,

$$
\max (x, y)=(x \underset{€}{\rightarrow} y) \underset{€}{\rightarrow} y,
$$

where $\underset{\mathrm{t}}{\rightarrow} \mathrm{y}$ is Eukasiewicz implication.

Proof. Consider disjunction operation $x \vee y=\max (x, y)$. Because $0 \leq x, y \leq 1$, using the arithmetical formula (8.4) for max, we have 


$$
\begin{aligned}
\max (x, y) & =\min \{1, \max (x, y)\}=\min \left\{1, \frac{x+y+|x-y|}{2}\right\} \\
& =\min \left\{1, \frac{2-1-1+x+2 y-y+|1-1+x-y|}{2}\right\} \\
& =\min \left\{1,1-\frac{1+(1-x+y)-|1-(1-x+y)|}{2}+y\right\} \\
& =\min \{1,1-\min (1,1-x+y)+y\}
\end{aligned}
$$

On the other hand, in $\succeq_{\aleph_{1}}$ disjunction is defined by(Disj.), i.e., by the formula

$$
x \vee y=(x \underset{€}{\rightarrow} y) \underset{Ł}{\rightarrow} y
$$

When we apply the evaluation rule of implication (Impl.) to the right side of the equation (8.9) we get the equation

$$
x \vee y=(x \underset{\mathrm{E}}{\rightarrow} y) \underset{\mathrm{E}}{\rightarrow} y=\min \{1,1-\min (1,1-x+y)+y\}=\max (x, y)
$$

by (8.8). Hence, the assertion (8.7) follows, and the proof is complete.

Of course, Łukasiewicz must have known the connection between maximum operation and his truth evaluation formula (Impl.) of the implication because without any knowledge about this, he would have not been sure that everything fits well together in his logic. But how he has inferred this is not known. Maybe, he has shown this in some special cases by truth tables with $n$ truth values where $n$ is finite.

The result of the proof of the formula (8.7) shows that from the join operation max of our algebra we deduce a formula that expresses the rule of Łukasiewicz' implication, and this formula is the truth value evaluation rule in $€_{\aleph_{1}}$. Hence, we have shown that from our algebra (6.7) it is possible to derive similar rules as the truth value evaluation rules in $€_{\aleph_{1}}$.

Hence, we may conclude our main result in a formal way:

Proposition 8.3. If the cases

1. $\neg x=1-x$;

2. $x \vee y=\max (x, y)$;

hold, then the other cases

3. $x \wedge y=\min (x, y)$;

4. $x \rightarrow y=\min (1,1-x+y)$;

5. $x \leftrightarrow y=(x \rightarrow y) \wedge(y \rightarrow x)=1-|x-y|$,

can be derived based on Zadeh algebra (6.7).

Proof. The case 3 follows from the case 2 by duality. (Actually, this operation already belongs to Zadeh algebra, and hence to $\succeq_{\aleph_{1}}$.) 
The case 4 follows from the case 2 by Prop. 8.2 as follows. When we consider the equation (8.10) in the proof of Prop. 8.2, we find two min-stuctures corresponding to the evaluation rule of implication, so that one of them is an inside part of the whole formula. If we denote the inner min-structure $\min (1,1-x+y)$ by $z$ then the outer min-structure is $\min (1,1-z+y)$, i.e., the min-structures are formally the same. The implication operations in (8.9) are situated in the same way. Hence, $\min (1,1-x+y)$ must be the evaluation rule of $x \underset{\mathrm{E}}{\rightarrow} y$, by (Impl.).

The case 5 is deduced as follows:

$$
\begin{aligned}
x \leftrightarrow y & =\min \{(x \rightarrow y),(y \rightarrow x)\} \\
& =\frac{(x \rightarrow y)+(y \rightarrow x)-|(x \rightarrow y)-(y \rightarrow x)|}{2} \\
& =\frac{\min (1,1-x+y)+\min (1,1-y+x)}{2} \\
& -\frac{\min (1,1-x+y)-\min (1,1-y+x)}{2} \\
& =\frac{4-2|x-y|-|-2 x+2 y-| x-y|+| x-y||}{4} \\
& =\frac{4-4|x-y|}{4}=1-|x-y| .
\end{aligned}
$$

Hence, the connectives of $\succeq_{\aleph_{1}}$ can be created by Zadeh algebra.

These cases are similar to the truth value evaluation rules for connected formulas in $\succeq_{\aleph_{1}}$. Hence, if we want to use algebraic approach for $\succeq_{\aleph_{1}}$ we need not necessarily to follow the mainstream described in Section 9 using the operations of MV-algebras for studying the connections between the connectives in $€_{\aleph_{1}}$.

However, in the next section, we give also a very brief description about the alternative approach starting from the definition of general MV-algebra. It is the mainstream in this research topic, but a circuitous route in the case of Łukasiewicz logic.

\section{The relationship between Łukasiewicz logic and MV-algebras}

We open another way a little for creating an algebra for Łukasiewicz logic. We adopt the definition and some properties of MV-algebras from Cignoli et. al. (Cignoli et al., 2000). Other sources are Bergmann (Bergmann, 2008) and Hájek (Hájek, 1998).

Definition 9.1. An $M V$-algebra is an algebra $\langle A, \oplus, \neg, 0\rangle$ with a binary operation $\oplus$, a unary operation $\neg$ and a constant 0 satisfying the following equations:

(MV1) $\quad x \oplus(y \oplus z)=(x \oplus y) \oplus z$

(MV2) $\quad x \oplus y=y \oplus x$

(MV3) $\quad x \oplus 0=x$

(MV4) $\neg \neg x=x$

(MV5) $\quad x \oplus \neg 0=\neg 0$

(MV6) $\neg(\neg x \oplus y) \oplus y=\neg(\neg y \oplus x) \oplus x$

A non-empty set $A$ is the universe of the MV-algebra $\langle A, \oplus, \neg, 0\rangle$. 
In particular, axioms (MV1) - (MV3) state that $\langle A, \oplus, 0\rangle$ is an abelian monoid.

Given an MV-algebra $A$ and a set $X$, the set $A^{X}$ of all functions $f: X \longrightarrow A$ becomes an MV-algebra if the operations $\oplus$ and $\neg$ and the element 0 are defined pointwise. It is obvious that the unit interval $[0,1]$ is an MV-algebra. The continuous functions from $[0,1]$ into $[0,1]$ form a subalgebra of the MV-algebra $[0,1]^{[0,1]}$.

On each MV-algebra $A$ we define the constant 1 and the operations $\odot$ and $\ominus$ as follows:

$$
\begin{gathered}
1 \stackrel{\text { def }}{=} \neg 0, \\
x \odot y \stackrel{\text { def }}{=} \neg(\neg x \oplus \neg y), \\
x \ominus y \stackrel{\text { def }}{=} x \odot \neg y .
\end{gathered}
$$

An MV-algebra is nontrivial is and only if $0 \neq 1$. The following identities are immediate consequences of (MV4):

(MV7) $\neg 1=0$,

(MV8) $\quad x \oplus y=\neg(\neg x \odot \neg y)$.

Axioms (MV5) and (MV6) can now be written as:

$\left(\mathrm{MV5}^{\prime}\right) \quad x \oplus 1=1$,

$\left(\mathrm{MV6}^{\prime}\right) \quad(x \ominus y) \oplus y=(y \ominus x) \oplus x$.

Setting $y=\neg 0$ in (MV6) we obtain

(MV9) $x \oplus \neg x=1$,

In the MV-algebra $\langle[0,1], \oplus, \neg, 0\rangle$ we have

$$
\begin{aligned}
& x \odot y=\max (0, x+y-1) \\
& x \ominus y=\max (0, x-y)
\end{aligned}
$$

Notation: Following common usage, we consider the $\neg$ operation more binding than any other operation, and the $\odot$ operation more binding than $\oplus$ and $\ominus$.

Consider the question about the connection between Łukasiewicz implication and operations in MV-algebra.

Given an MV-algebra $\langle A, \oplus, \neg, 0\rangle$ and a set $X$, the set

$$
A^{X}=\{f \mid f: X \longrightarrow A\}
$$

becomes an MV-algebra if the operations $\oplus$ and $\neg$ and the element 0 are defined pointwice (Cignoli et al. (Cignoli et al., 2000), p. 8). To define 0 pointwice means here that the result is a constant function $0: x \mapsto 0$ for any $x$ in the universe of that algebra.

Further, Łukasiewicz implication

$$
x \rightarrow y \stackrel{\text { def }}{=} \min \{1,1-x+y\}
$$


can be expressed in MV-algebra in the form (cf. Cignoli et al. (Cignoli et al., 2000), p. 78)

$$
x \rightarrow y=\neg x \oplus y
$$

whence,

$$
x \oplus y=\neg x \rightarrow y .
$$

The equation (9.6) shows that between Łukasiewicz implication ant the operation $\oplus$ there is a similar connection as in S-implications which are defined by means of disjunction. But $\oplus$ is not disjunction in Łukasiewicz logic. In many cases $\oplus$ is interpreted as disjunction, but defined on the unit interval it gives different values as Łukasiewicz disjunction operation max. However, it is possible to define the operations max and min by means of the operations of MV-algebra, but then the result usually is a logic with additional operations having no reasonable interpretations ( $c f$. for example, the logic Fuzzy ${ }_{L}$ in Bergmann's book (Bergmann, 2008). Some comments on Fuzzy ${ }_{L}$ is given in (Mattila, 2010)).

Wajsberg created an algebra, called by Wajsberg algebra (W-algebra, for short) which is know to serve an algebraic approach to Łukasiewicz infinite-valued logic. The following lemma ( $c f$. Cignoli et al. (Cignoli et al., 2000), p. 83) gives the connection between Wajsberg algebras and MV-algebras.

Lemma 9.1. Let $A$ be an $M V$-algebra, and put $x \rightarrow y \stackrel{\text { def }}{=} \neg x \oplus y$ and $1 \stackrel{\text { def }}{=} \neg 0$. Then $\langle A, \rightarrow, 1\rangle$ is $a$ W-algebra.

The binary operation of $\mathrm{W}$-algebra is implication operation. In this algebra a unary operation is $\neg$ because it is needed to create the unit element 1 . The zero element 0 belongs to this algebra because it implies the unit element by means of negation. Hence, the algebra is in a suitable form according to Łukasiewicz logic. Now, we have counterparts of the primitive connectives of Łukasiewicz logic as the operations of $\mathrm{W}$-algebra. The other connectives can be created in the similar way in $\mathrm{W}$-algebra as Łukasiewicz has introduced them.

One consequence from this consideration is that in MV-algebras the operations max and min can be created by the operations of $\mathrm{W}$-algebra, i.e., by the primitive connectives of Łukasiewicz logic by means of the evaluation rule of (9.6).

\section{Conclusion}

The main problem we considered here is to find connections between standard fuzzy sets and Łukasiewicz logic $\succeq_{\aleph_{1}}$ and to find a suitable algebra for it, especially, because the primitive connectives are negation and implication. In De Morgan algebras the counterparts for the logical connectives disjunction, conjunction, and negation appear as the algebraic operations. It cannot immediately be seen how Łukasiewicz implication, that belongs to the primitive connectives, are derived from the disjunction $(\max )$ and negation $(\neg x=1-x, x \in \mathbb{I})$. We have done it here using a special De Morgan algebra, namely, Zadeh algebra. Hence, the connection between standard fuzzy sets and Łukasiewicz logic $Ł_{\aleph_{1}}$ becomes clear. The key result, where Łukasiewicz implication is derived algebraically from disjunction and negation, is given in Proposition 8.3.

Kleene's logic is considered because of its close connection to standard fuzzy sets, already motivated by Zadeh. The sections 2, 3, 4, 6, and 8 gives the method we have used for creating our results. 
The section 9 tells very briefly how the others consider this topic. That way is different and alternative to ours. MV-algebra is quite general, and many algebras, like Boolean algebras and also De Morgan algebras belong to its scope. The reader may become familiar with this topic, for example, by reading the Bergmann's, Cignoli's et. al, and Hájek's books mentioned in References. A lot of other material is available, too.

Our alternative way we have considered the topic here, is not totally new, because these things are considered in (Mattila, 2004), (Mattila, 2005), and (Mattila, 2010), but our key result, Proposition 8.3 is. Proposition 8.2 is the core of this result. It makes the connection of max operation and Łukasiewicz inmplication clear by means of Zadeh algebra. Using the expressions (8.4) for the operations max and $\mathrm{min}$ is usually not used in general, but it makes the consideration easy. As we can see from the used references, De Morgan algebras have already been well known relatively long time having, in the long run, different alternative names, like "quasi Boolean algebras", and "soft algebras".

H. Rasiowa has considered implicative algebras and implication algebras in her book ((Rasiowa, 1974)). Hence, the future research policy may be based on these algebras. Also, the connections between implicative/implication algebras and De Morgan algebras or $\mathrm{MV}$-algebras restricted to many-valued or modal logics are included to the future research.

\section{References}

Bergmann, M. (2008). An Introduction to Many-Valued and Fuzzy Logic, Cambridge University Press, New York, Melbourne, Madrid,Cape Town, Singapore, São Paulo, Delhi.

Cignoli, L. \& D'Ottaviano, M. \& Mundici, D. (2000). Algebraic Foundations of Many-valued Reasoning, Kluwer Academic Publishers, Dordrecht, Boston, London.

Hájek, P. (1998). Metamathematics of Fuzzy Logic, Kluwer Academic Publishers, Dordrecht, Boston, London.

Lowen, R. (1996). Fuzzy Set Theory. Basic Concepts, Techniques and Bibliography, Kluwer Academic Publishers, Dordrecht, Boston, London.

Mattila, J. K. (2004). Zadeh algebras as a syntactical approach to fuzzy sets, Current Issues in Data and Knowledge Engineering, Springer-Verlag, Warszawa, Poland, pp. 343-349.

Mattila, J. K. (year 2005). On łukasiewicz modifier logic, Journal of Advanced Computational Intelligence and Intelligent Informatics Vol. 9(No. 5): 506-510.

URL: www.fujipress.jp

Mattila, J. K. (2009). Many-valuation, modality, and fuzziness, in Seising R. (ed.), Views on Fuzzy Sets and Systems from Different Perspectives. Philosophy and Logic, Criticisms and Applications, Springer-Verlag, Berlin, Heidelberg, pp. 271-300.

Mattila, J. K. (2010). On Łukasiewicz' infinite-Valued logic and Fuzzy ${ }_{L}$, in, KES 2010 Knowledge-Based Intelligent Information and Engineering Systems, Part IV, LNAI 6279, Springer-Verlag, Berlin, Heidelberg, pp. 108-115.

Negoită, C. V. \& Ralescu, D. A., (1975). Applications of Fuzzy Sets to Systems Analysis, Birkhäuser, Basel, Stuttgart.

Rasiowa, H. (1974). An Algebraic Approach to non-classical Logics, North-Holland.

Rescher, N. (1969). Many-Valued Logic, McGraw-Hill, New York, St. Louis, San Francisco, London, Sydney, Toronto, Mexiko, Panama.

Zadeh, L. A. (1965). Fuzzy Sets, Information and Controll 8. 


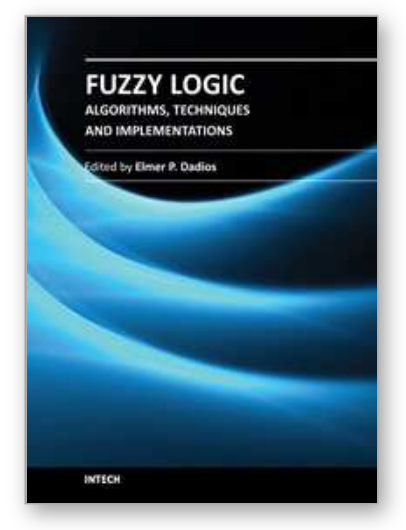

\author{
Fuzzy Logic - Algorithms, Techniques and Implementations \\ Edited by Prof. Elmer Dadios
}

ISBN 978-953-51-0393-6

Hard cover, 294 pages

Publisher InTech

Published online 28, March, 2012

Published in print edition March, 2012

Fuzzy Logic is becoming an essential method of solving problems in all domains. It gives tremendous impact on the design of autonomous intelligent systems. The purpose of this book is to introduce Hybrid Algorithms, Techniques, and Implementations of Fuzzy Logic. The book consists of thirteen chapters highlighting models and principles of fuzzy logic and issues on its techniques and implementations. The intended readers of this book are engineers, researchers, and graduate students interested in fuzzy logic systems.

\title{
How to reference
}

In order to correctly reference this scholarly work, feel free to copy and paste the following:

Jorma K. Mattila (2012). Standard Fuzzy Sets and some Many-Valued Logics, Fuzzy Logic - Algorithms, Techniques and Implementations, Prof. Elmer Dadios (Ed.), ISBN: 978-953-51-0393-6, InTech, Available from: http://www.intechopen.com/books/fuzzy-logic-algorithms-techniques-and-implementations/standard-fuzzysets-and-some-many-valued-logics

\section{INTECH}

open science | open minds

\section{InTech Europe}

University Campus STeP Ri

Slavka Krautzeka 83/A

51000 Rijeka, Croatia

Phone: +385 (51) 770447

Fax: +385 (51) 686166

www.intechopen.com

\section{InTech China}

Unit 405, Office Block, Hotel Equatorial Shanghai

No.65, Yan An Road (West), Shanghai, 200040, China

中国上海市延安西路65号上海国际贵都大饭店办公楼 405 单元

Phone: +86-21-62489820

Fax: +86-21-62489821 
(C) 2012 The Author(s). Licensee IntechOpen. This is an open access article distributed under the terms of the Creative Commons Attribution 3.0 License, which permits unrestricted use, distribution, and reproduction in any medium, provided the original work is properly cited. 